Problems (S. 174) überhaupt nicht erörtert werden. Indessen wäre hier - wie auch an einigen anderen Stellen - über allgemeine Erwägungen zur völkerrechtlichen Ächtung hinaus substantieller Fortschritt wohl nicht ohne Einbeziehung des neuesten Diskussionsstandes im Arbeitskollisionsrecht zu erzielen gewesen ${ }^{8}$. Dieses blieb aber (leider) bewußt aus der Untersuchung ausgespart (S. 204).

Insgesamt ein nützliches, engagiert geschriebenes Buch, dessen Stärke in der Darstellung tatsächlicher Grundlagen des internationalen Wirtschaftssystems liegt. Daß die sich in diesem Bereich auftürmenden Rechtsfragen hier und dort unbedingt weiterer Bearbeitung zugänglich und bedürftig sind, tut dem positiven Urteil keinen Abbruch: An dogmatisch interessierten Juristen herrscht namentlich im deutschsprachigen Raum kein Mangel, eher schon an solchen mit einem ausgeprägten Blick für wirtschaftliche und politische Fakten.

Herbert Kronke

\title{
Annuaire de l'Afrique du Nord, XVI, 1977
}

publié par le Centre de Recherches et d'Etudes sur les Sociétés Méditerranéenes, Aix-enProvence, France, Editions du Centre National de la Recherche Scientifique, Paris, 1978, $1348 \mathrm{~S}$.

Die seit 1962, dem Jahr der Unabhängigkeit Algeriens, regelmäßig erscheinenden Jahrbücher des renommierten französischen Instituts ,,Centre de Recherches et d'Etudes sur les Sociétés Méditerranéennes" der Universität in Aix-en-Provence sind das Standardwerk für jeden, der sich mit dem aktuellen Geschehen in den Maghrebländern beschäftigt. Die sehr umfangreichen und umfassenden Bände erscheinen im allgemeinen etwa 1 1/2 Jahre nach dem behandelten Jahr, ein Zeitraum, der für die textliche Bearbeitung und technische Herstellung kaum kürzer sein kann. So kam der letzte vorliegende und hier zur Besprechung stehende Band im Herbst 1979 heraus, greift aber auch schon über das eigentliche Jahr hinaus. So wurden im Band 1977 auch schon Ereignisse von 1978 einbezogen.

Die Jahrbücher gliedern sich in sechs Abschnitte:

\section{Studien}

Hier werden Abhandlungen, meist unter einem gemeinsamen Thema, gebracht, so im vorliegenden Band die institutionellen Reformen und die Legitimation der Staatsgewalt in den Maghrebländern. Bei der Behandlung werden die vier größeren Maghrebländer Algerien, Libyen, Marokko und Tunesien einzeln berücksichtigt. Die den Maghreb als Ganzes betreffenden Artikel folgen besonders. Das Westsahara-Problem erscheint unter ,,Aktuelle Fragen".

\section{Chronik des Jahres}

Dieser Abschnitt teilt sich auf in: diplomatische, politische, wirtschaftliche, soziale und kulturelle Chronik. Darin werden die aktuellen Entwicklungen in den einzelnen Maghrebländern jeweils besonders dargestellt. Im vorliegenden Band gibt es dazu für Mauretanien einen zusammengefaßten Jahresbericht. Es folgt dann eine genaue, datenmäßige Aufstellung (Chronologie) über alle relevanten Ereignisse in den Maghrebländern und im Gesamtmaghreb. Dazu geben eine Liste der Abkommen, Verträge, sonstigen Vereinbarungen und Erklärungen sowie eine Gesetzgebungsübersicht die notwendigen sachlichen Ergänzungen.

8 Vgl. Birk, BerDGesVR 18 (1978) 263 ff. und meine Bespr., VRU 13 (1980) $61 \mathrm{ff.}$ 


\section{Dokumente}

Hier werden wichtige Gesetze, grundsätzliche Erklärungen, Reden, Kommuniqués und Regierungslisten im Wortlaut abgedruckt, darunter im vorliegenden Band auch interessante Dokumente zum Sahara-Konflikt.

\section{Kunst und Literatur}

In diesem Abschnitt werden Artikel zu aktuellen Entwicklungen gebracht, so im vorliegenden Band zum ,Film in Algerien“ und zum „Theater in Tunesien“.

\section{Wissenschaft}

In Aufsätzen werden aktuelle Themen aus Wissenschaft und Forschung behandelt sowie Berichte über wissenschaftliche Konferenzen und Kolloquien zu Themen des Maghreb und Islam erstattet. Verwiesen sei besonders auf einen Beitrag zur Berber-Sprache und -Literatur.

\section{Bibliographie}

Dieser Abschnitt bringt zunächst zahlreiche Besprechungen neu erschienener Bücher, aufgegliedert nach: Allgemeines - Recht, Wirtschaft, Politik - Gesellschaft - Literatur. Es folgt eine systematische Aufstellung (Bibliographie) aller Neuerscheinungen des Jahres 1977, aufgeteilt nach europäischen Sprachen und Arabisch (mit französischer Úbersetzung von Verfasser und Titel) nebst Index der Verfasser:

Der vorliegende XVI. Band des Annuaire (AAN) verdient besonderes Interesse, da in ihm ein Zeitraum behandelt wird, in dem sich wichtige verfassungsrechtliche Entwicklungen in den Maghrebländern vollzogen, und zwar traten diese Länder nach den ersten Verfassungsschritten unmittelbar nach ihrer Unabhängigkeit aufgrund der seitdem gewonnenen Erfahrungen nunmehr in eine neue Konstitutionalisierungsphase ein, die eine Konsolidierung der politischen Verhältnisse und eine Stärkung der nationalen Eigenheit darstellt. Das gilt vornehmlich für die Kernländer Marokko, Algerien und Tunesien, während Libyen unter seinem eigenwilligen Führer Ghaddafi eher noch weiter experimentiert.

Marokko erhielt zwar schon 1972 eine neue Verfassung, die aber erst 1977 endgültig zur Anwendung kam. Beginnend im November 1976 auf Kommunal- und Munizipalebene wurden Wahlen nach und nach auf Provinzial-, Präfektoral-, Professional- und Legislativebene durchgeführt, womit der dritte parlamentarische Versuch in Marokko einsetzte. Die Parlamentswahlen vom Juni 1977 brachten bei $81 \%$ Wahlbeteiligung einen klaren Sieg der Unabhängigen, die eine neue Generation von 35- bis 40jährigen mit höherer Schulbildung und in selbständigen Berufen oder im öffentlichen Dienst repräsentieren. Sie stützen sich auf eine heterogene Wählerbewegung, die von einem starken monarchistischen Schwung getragen wird, der sich im Kampf um die Westsahara entzündete und im ,,Grünen Marsch“ seinen solidarischen Ausdruck fand. Auch die traditionelle Istiqlal-Partei bestätigte sich als politische Kraft und nimmt an der Regierung teil. Der König bekannte sich bei der Parlamentseröffnung am 14. Oktober 1977 zur Demokratie, zum Pluralismus und zur Liberalisierung des politischen Lebens. Die Legitimation der Staatsgewalt zur Vertretung der sozialen Kräfte hat sich gefestigt, und die nunmehr ausgefüllte Verfassung hat Kraft gewonnen. - Der Ablauf der Wahlen, die Erneuerung des politischen Lebens, die politischen Parteien, die institutionellen Mechanismen, die Autonomie von Regierung und Parlament, die Aussichten auf Modernisierung des Staatsapparates werden in den Studien des AAN eingehend behandelt. Dabei heißt es zum Schluß: Die jüngsten konstitutionellen und politischen Entwick- 
lungen, so bedeutend sie sein mögen, werden nebensächlich bleiben, wenn die Institutionen und politischen Kräfte nicht tatsächlicher Ausfluß der sozialen Wirklichkeit des Landes werden.

Algerien hat sich 1976-1978 mit einem beachtlichen Verfassungswerk eine solide Staatsgrundlage geschaffen. Der ersten Verfassung nach der Unabhängigkeit von 1962 war nur ein kurzes Leben beschieden. Sie wurde sehr schnell vom damaligen Präsidenten Ben Bella durch Ausnahmerecht beiseitegeschoben. Als 1965 Boumediène die Macht ergriff, regierte er aufgrund Revolutionsrechts, allerdings in maßvoller und wohlüberlegter Weise. Langsam reiften seine Ideen und Pläne für das neue Algerien. 1976 legte er in einer ,,Charte Nationale" die Ideologie und verfassungsrechtlichen Grundlagen des neuen Staates dem Volk zur Erörterung vor. Nach lebhafter öffentlicher Diskussion wurden entsprechende Änderungen und Ergänzungen vorgenommen. Der neuformulierte Wortlaut wurde zur Volksabstimmung gestellt und am 27. Juni 1976 mit großer Mehrheit angenommen. Die daraufhin entworfene Verfassung fand ebenfalls durch Volksabstimmung am 19. November 1976 mehrheitliche Zustimmung. Es folgten alsbald die allgemeine Wahl Boumediènes zum Präsidenten der Republik und am 25. Februar 1977 die Wahlen zur Nationalversammlung. Die Studien im AAN behandeln ausführlich diese Vorgänge und untersuchen eingehend die Charta und die Verfassung sowie die Bedeutung des durch sie eingeführten Sozialismus. Hervorgehoben wird der ideologisch revolutionäre Charakter des algerischen politischen Regimes. Das Volk der sozialistischen Revolution ist untrennbar das Volk der nationalen Revolution, der arabischen, der drittweltlichen Revolutionen und aller Revolutionen, die noch aus der algerischen Revolution entstehen können.

Schwierigkeiten bereitet nur die Erneuerung und Belebung der Einheitspartei, Front de Libération Nationale (FLN), die Avantgarde und Rückgrat der Nation sein soll. Der für Mitte 1978 vorgesehene, stimulierende Parteikongreß fand nicht statt. Dagegen wurden die Vorläufer-Kongresse der großen Massenorganisationen, wie UGTA (Arbeiter), UNPA (Bauern), UNFA (Frauen), UNJA (Jugend), wie vorgesehen, 1978 abgehalten. - Seine Bewährungsprobe bestand das Verfassungswerk aber, als Ende Dezember 1978 der Präsident Houari Boumediène unerwartet starb und Anfang 1979 der Machtübergang auf seinen Nachfolger Bendjedid Chadli sich reibungslos vollzog und endlich auch der Parteikongreß zustande kam. Algerien hat sich damit als weitgehend konsolidiert erwiesen.

Tunesien hat 1976 die erste, substantiellere Revision seiner Verfassung von 1959 erfahren. Bedeutung und Umfang dieser Verfassungs reform werden in einer Studie im vorliegenden AAN von 1977 ausführlich dargestellt und kritisch gewürdigt.

Die Verfassung von 1959 war ganz auf die Person des Befreiers und ,,Combattant Suprême“ Habib Bourguiba zugeschnitten, der das Land souverän und patriarchalisch regierte, obwohl das Land sich als republikanisch und sozialistisch bezeichnete. Daran hat sich auch jetzt grundsätzlich nichts geändert; jedenfalls zeigt sich keine Hinwendung zur Liberalisierung und Demokratisierung des Systems. Die Revision der Verfassung von 1976 stellt den Abschluß eines Prozesses der Anpassung dar, der 1969 einsetzte, als dem kränkelnden Bourguiba die Zügel der Regierung etwas entglitten und er sich durch seinen ,,K ronprinzen“ und Superminister Ben Salah getäuscht fühlte. Der älter und leidend gewordene Präsident wollte aber die Regierung nicht aus der Hand geben, er wollte sich nur von seinen Aufgaben entlasten, ohne jedoch die Kontrolle des Staates zu verlieren. Daraus nun ist ein ausgeklügeltes System geworden, das einzigartig und nur durch die Person Bourguibas und die.ihm entgegengebrachte Loyalität zu verstehen ist. Er ist Chef des Staates und Chef der Einheitspartei. 
Er bestimmt über alles, und alle Institutionen sind auf ihn ausgerichtet. Die Regierung erhält ihre Gewalt von ihm und ist nur ihm verantwortlich. Aufgrund von Aufgabendelegation erhält der Ministerpräsident praktisch politisches Gewicht, das um so größer wird, als der Präsident die Zügel lockerläßt. Die Legislative, d. h. die Nationalversammlung, hat grundsätzlich die Gesetzgebungsgewalt - aber nicht ausschließlich und unbeschränkt, da dem Präsidenten weitgehende Vollmachten verbleiben und auch der Regierung dabei eine wichtige Rolle zufällt. Das einzig Positive ist die Sicherung des Verfahrens der Nachfolge beim Tode des auf Lebenszeit gewählten Präsidenten Bourguiba. Allerdings wird nach seinem Ende dieses einzigartige, personalbezogene Präsidialsystem nicht fortbestehen können. Es wird zweifellos im politischen und sozialen Leben Tunesiens zu grundlegenden Veränderungen kommen, deren Vorzeichen gerade im Jahre 1977 sehr deutlich wurden, wie in der politischen Chronologie des AAN aufgezeigt wird.

Das Jahr war gekennzeichnet durch den ,,Sozialpakt“", der am 19. Januar 1977 von den ,,Sozialpartnern“", d. h. der Regierung, dem Politbüro der Partei (PSD), dem Gewerkschaftsbund (UGTT), dem Industrie-, Handels- und Gewerbeverband (UTICA) und dem Bauernverband (UNA) abgeschlossen wurde und zu einer Gesundung des Sozialklimas führen sollte, das 1976 durch eine Reihe von Streiks und Forderungen stark gestört worden war. Aber schon ab Mitte des Jahres gab es neue Zeichen einer heftigen Verschlechterung des sozialen und politischen Klimas. Verschiedene politische Gruppierungen rührten sich, so das ,, Mouvement de l'Unité Populaire“ (MUP) des Ahmed Ben Salah und die ,,Liberalen“ oder ,,Sozialdemokraten" um Ahmed Mestiri, und forderten eine Politik der Offnung und Anerkennung des sozialen und politischen Pluralismus. Die Idee der ,Dezentralisierung“ wird zum Zauberwort. Man sieht darin die Antinomie Macht - Freiheit, die Anti-Gewalt, ein liberales Regierungsprinzip und die Humanisierung für das demokratische System. Besonders in der Gewerkschaftsbewegung findet die Dezentralisierung großen Widerhall. - Die soziale und politische Spannung im Lande wächst zunehmend. Der Generalsekretär der UGTT, Habib Achour, erklärt seinen Rücktritt als Mitglied des Politbüros und des Zentralkomitees der PSD. Der Nationalrat der UGTT kritisiert die Regierung und bestätigt nicht nur den Prozeß der Dezentralisierung der UGTT, sondern zog daraus noch Folgerungen für das politische System. Es kommt am 26. Januar 1978, dem ,,Schwarzen Donnerstag“ zu blutigen Zwischenfällen unter Einsatz der Armee und Erklärung des Staatsnotstandes und damit auch zum Ende des Sozialpaktes. - Tunesien befindet sich in einem sozialen und politischen Wandlungsprozeß, der auch vor der Verfassung von 1976 keinen Halt machen wird.

In Libyen liegen die Dinge anders als in den vorgenannten Maghrebländern. Libyen ist im Ganzen rückständiger, ursprünglicher, volksärmer, mehr dem Traditionellen verbunden und der arabisch-islamischen Welt zugewandt. Dementsprechend sind auch unsere Kenntnisse und Informationen über das Land, sein Denken und Fühlen und seine Entwicklung geringer. Deshalb ist sehr zu begrüßen, daß in einer Studie im vorliegenden Band des AAN 1977 die Entwicklung der politischen Institutionen in Libyen von der Revolution im September 1969 über 10 Jahre bis 1978 dargestellt wird. Aber auch dann bleibt noch manches dunkel, zumal die Sprunghaftigkeit des Präsidenten Ghaddafi uns verwirrt. Erstaunlich viel ist in der verhältnismäßig kurzen Zeit passiert.

Die Innenpolitik Ghaddafis und damit auch der Aufbau der Institutionen ist eng verknüpft mit Ghaddafis außenpolitischen Plänen und panarabischen Ideen. Aber auch hier hat er bemerkenswerte Schwenkungen vorgenommen.

In einer ersten institutionellen Phase von 1969-1974 erläßt er eine vorläufige Verfassung (11. 12. 1969) nach ägyptischem Vorbild. Aber schon bald sucht er näheren Kontakt zum Volk. 
Seine Volksbegegnungen waren das Vorspiel für das System direkter Demokratie von 1977. Aber vorher kam es 1971 zur Vereinigung mit Ägypten durch die gemeinsame ,,Union Socialiste Arabe“" (USA), eine Verbindung, die aber schon 1973 scheiterte. Am 15. April 1973 startete Ghaddafi die Idee der ,,Volksrevolution“. Ein Jahr später zog er sich unerwartet zu einer ideologischen Besinnung zurück. Das brachte einen Umschwung: Der 1. Band seines Grünbuches erscheint, die Volksrevolution wird in Gang gesetzt und die zweite institutionelle Phase beginnt (1974-1978), in der die USA und der Revolutionsrat schließlich aufgelöst werden (1977). Wichtige Etappen waren der 2. Kongreß der libyschen USA im November 1974, dem die Aufgabe zufiel, den Ubergang zur Volksrevolution herzustellen und diese zu orientieren und dann die Rede Ghaddafis vom 27. April 1975, in der er eine genauere Darstellung des neuen Systems der Volksgewalt gab, wobei er besonderen Akzent auf den Sozialismus legte. Es folgte langsam die Einsetzung neuer Institutionen.

Ein 1. Allgemeiner Volkskongreß (AVK) trat gemäß der neuen Formel im Januar 1976 zusammen und brachte in seinen Beschlüssen erstmals die ,, direkte Volksdemokratie" zur Geltung. Ein 2. Kongreß folgte im November desselben Jahres. Auf Wunsch Ghaddafis sollte er eine ,,Proklamation der Errichtung der Volksgewalt“, eine Art historischer Erklärung wie die Proklamation der Menschenrechte annehmen. Dies geschah aber erst auf einem außerordentlichen Kongreß im März 1977, an dem u. a. auch Fidel Castro teilnahm. Die Annahme der Proklamation bedeutete die Anerkennung der programmatischen Rede Ghaddafis vom April 1975 und seines Grünbuches. Ihr folgten drei Dekrete: 1. Ernennung Ghaddafis zum Generalsekretär des AVK, 2. Beibehaltung des Revolutionsrats unter der neuen Bezeichnung Generalsekretariat des AVK und Ernennung von vier Mitgliedern und Ghaddafi, 3. Ernennung einer Regierung unter der Bezeichnung „Allgemeiner Volksausschuß“.

Anfang 1978 gibt Ghaddafi sein 2. Grünbuch heraus, in dem er neue Perspektiven für den libyschen Sozialismus darlegt und grundlegende Veränderungen der libyschen Gesellschaft ankündigt. Die neue sozialistische Gesellschaft bedeutet für Ghaddafi nichts anderes als die Rückkehr zum Naturrecht. Sie müßte nach seiner Auffassung auf das Verschwinden des Gewinns und des Geldes hinauslaufen, wofür jedoch noch Entwicklungen notwendig seien. So rief Ghaddafi am 1. Mai 1978 die Libyer auf, die Leitung ihrer Betriebe selbst zu übernehmen. Die Nationalisierung der Wohnungen erfolgte am 6. 5. 1978. Man kann eine wirkliche Revolution beobachten.

Abschließend meinen die Verfasser der Studie: Unbestreitbar liegt die Macht in Libyen fast ganz auf den Schultern von Ghaddafi. Das will aber nicht sagen, daß es sich um eine Diktatur handelt. Ghaddafi kann nicht machen, was er will, wie der Kongreß von November 1976 zeigte. Nichtsdestoweniger ist er es, der die Ideologie und als Folge davon auch die Dinge bestimmt. Das Regime hängt an seiner Person, an seinem Prestige und Schicksal. - Allerdings eine sehr gebrechliche Grundlage, zumal wenn man bedenkt, daß es doch eine gewisse innere und äußere Opposition gibt. Hierzu macht der politische Chronist im AAN 1977 nähere Angaben. Man wird mit weiteren Ủberraschungen rechnen müssen. Man denke nur an Ghaddafis jüngste politische Aktionen der ,,Vereinigung Libyens mit Syrien“ Ende 1980 und der wohl nur als Annexion zu bezeichnenden ,,Vereinigung mit dem Tschad“ um die Jahreswende 1980/1981.

Es wäre zu wünschen, daß die von den Herausgebern des AAN angekündigte, angestrebte Verkürzung der Erscheinungsfrist des Jahrbuches wirklich erreicht wird, um die Aktualität des Werkes noch zu erhöhen. Tatsächlich ist der Band XVII des AAN für 1978 inzwischen erschienen. Er wird in einer der nächsten Ausgaben dieser Zeitschrift besprochen werden. Gerhard Moltmann 